\title{
Test Analysis of Serial Communication Extension in Mobile Nodes of Participatory Sensing System
}

\author{
Xinqiang Tang $^{1}$, Huichun Peng ${ }^{2}$ \\ ${ }^{1}$ School of Physical Education, Jiangxi Institute of Fashion Technology, Nanchang, 330201, China \\ ${ }^{2}$ School of Physical Education, Jiangxi University of Technology, Nanchang, 330098, China \\ xinqiang_tang@yeah.net
}

Keywords: Mobile node, Visual system, Sports projects, Logical judgment, Serial port expansion, Participatory.

\begin{abstract}
Mobile node distribution prediction is participatory sensing system to carry on the key effective data collection and message forwarding, this paper presents a mobile node distribution algorithm based on social relations, and the algorithm uses multi node communication interactive evaluation way of rural sports project, realizing the comprehensive analysis of the project rationality. At the same time, using logical judgment matrix carries out modeling for the sensor and using programming way allocates the wireless sensor nodes cache, which can obtain serial port expansion circuit and physical platform. Finally, through the wireless communication experiment, this paper obtains the delay characteristic curve and the analysis accurate rate curve of data transmission, which provide a new communication and evaluation system for the development of rural sports.
\end{abstract}

\section{Introduction}

Participatory sensing appears a perception technology in recent years, it is also known as the center of human perception. Participatory sensing uses a variety of data acquisition equipment and intelligent mobile phone of integrated specific sensor, which carry out interactive or autonomous acquisition, classification, transmission and analysis for human social status information, and then making a smart decision, they provide a service for human life and social activities [1-3]. Using participatory sensing systems, and combined with wireless sensor network nodes distribution, this paper designs the evaluation process of the rural sports project, the overall design framework and ideas are shown in Figure 1.

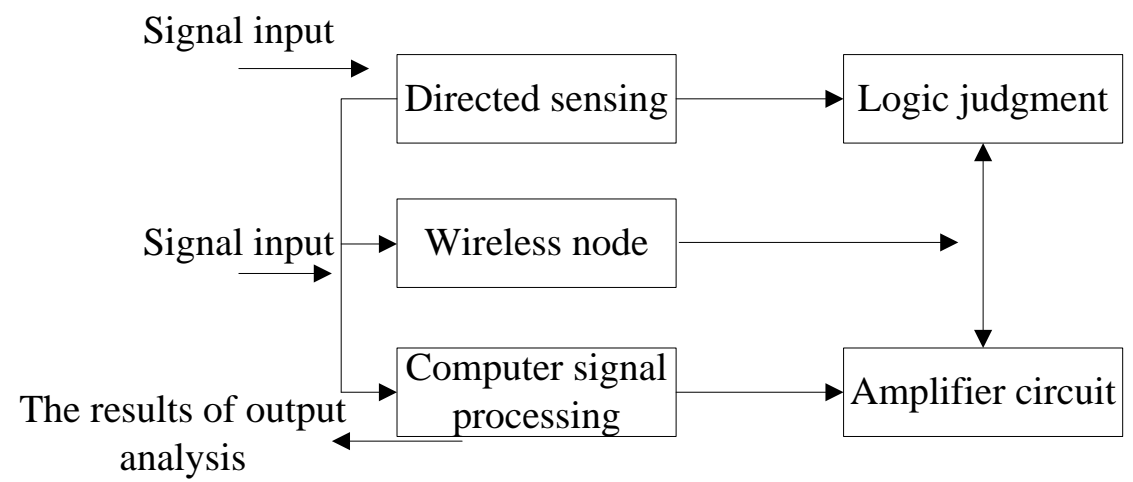

Fig. 1 Design of participatory sensing system

Figure 1 shows the overall design framework of participatory sensing system. Based on the directed perception principle, this paper designs the directional sensing mathematic model of wireless network coverage to improve the coverage of wireless nodes, and then using logical judgment function realizes the directional condition of signal transmission, finally using the amplification circuit makes the signal into the computer to carry on information processing, the results of the analysis will be eventually outputted. 


\section{Design of the Directional Adjustable Effective Perception Wireless Sensor Network Mathematical Model}

Participatory sensing system is mainly on the basis of multi node wireless communication way, the evaluation results of different evaluators carry out square summary, and then to carry on comprehensive evaluation or the rationality analysis of the sports [4,5]. Because of having more nodes participating evaluation, this paper designs directional adjustable directional sensing model to improve the wireless network coverage as shown in Figure 2.

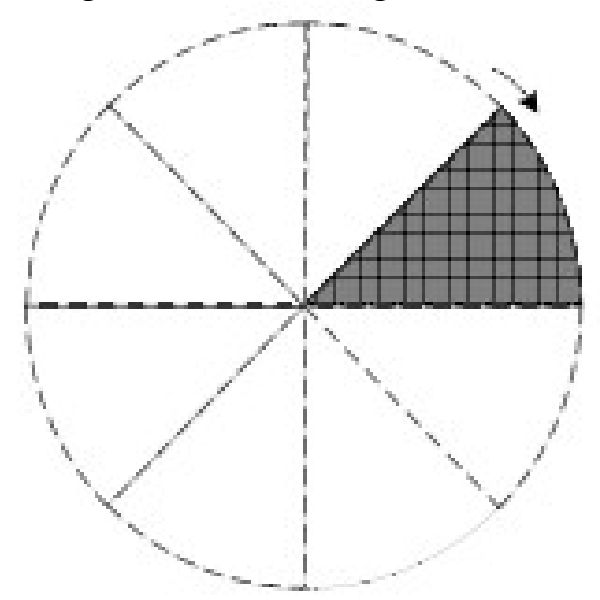

Fig.2: The directional adjustable sensing model

Directional sensing nodes can adjust the switch to the perception of different direction and each direction can cover the sensing range of limited angle, making the multi project analysis of participatory sensing analysis more accurate [6-8]. First, assume that there are the two variables $A=\left\{x_{1}, x_{2}, \cdots, x_{m}\right\}$ and $B=\left\{y_{1}, y_{2}, \cdots, y_{n}\right\} ; R$ is directional vector from $A$ to $B$. The relationship matrix is assumed

$$
M_{K}=\left[k_{i j}\right]_{m \times n} .
$$

Among them,

$$
k_{i j}=\left\{\begin{array}{ll}
1 & \text { if }<x_{i}, y_{j}>\in R \\
0 & \text { if }<x_{i}, y_{j}>\notin R
\end{array} .\right.
$$

Hypothesis

$$
\begin{aligned}
& A=\{1,2,3,4\} \\
& K=\{<1,1>,<1,2>,<2,3>,<2,4>,<4,2>\} .
\end{aligned}
$$

According to the logical relationship between nodes, we can give the direction relation matrix.

$$
M_{k}=\left[\begin{array}{llll}
1 & 1 & 0 & 0 \\
0 & 0 & 1 & 1 \\
0 & 0 & 0 & 0 \\
0 & 1 & 0 & 0
\end{array}\right] .
$$

The logical judgment of node signal can be used to the mapping to represent, if any $x, y \in Z_{1}$, the directional relationship can be represented by

$$
\mu(x 0 y)=\mu(x) * \mu(y) \text {. }
$$

In the relationship of using homomorphism relation, we can use programming way to adjustable sensor allocation cache, in which the main program is as follows [9-11]:

Typedef struct \{

Void *memAddr;

Void *memFreeList;

INT32 memBlkSize; 
INT32 memNBlks;

INT32 memNFree;

\}MEM_CTL;

MEM_CTL *memCreate(void *addr, INT32U nblks, INT32U blksize,।

INT8U *err);

Void *memGet(MEM_CTL *pmem, INT8U *err);

INT8U memPut(MEM_CTL *pmem, void *pblk);

\section{Design of Rural Sports Rationality Participatory Sensing Systems}

In order to verify the effectiveness and reliability of directional sensing wireless sensor mathematical model proposed in second part, this paper designs participatory wireless sensor network node distribution using the rationality analysis of rural sports, and designs serial port expansion circuit and physical platform, wherein the serial port expansion circuit are as shown in Figure 3.

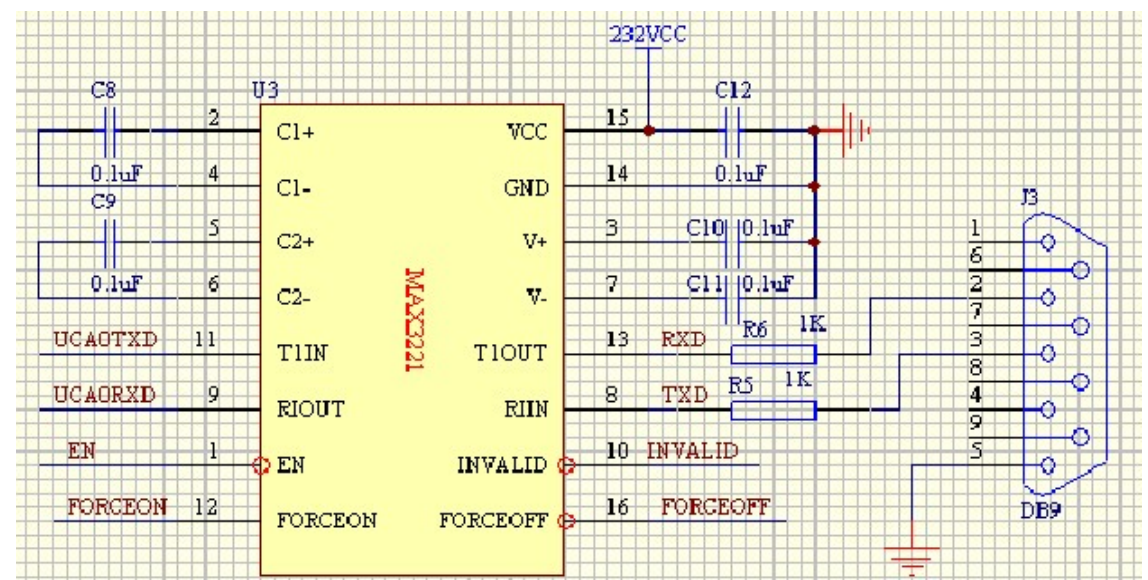

Fig.3: Serial port expansion circuit

In this paper, the use of serial port expansion circuit is MSP430 chip, it integrates ADC module, in which the MSP430FG4618 integrates 12 bit precision analog-to-digital conversion module ADC12, and it can be used for communication with PC.

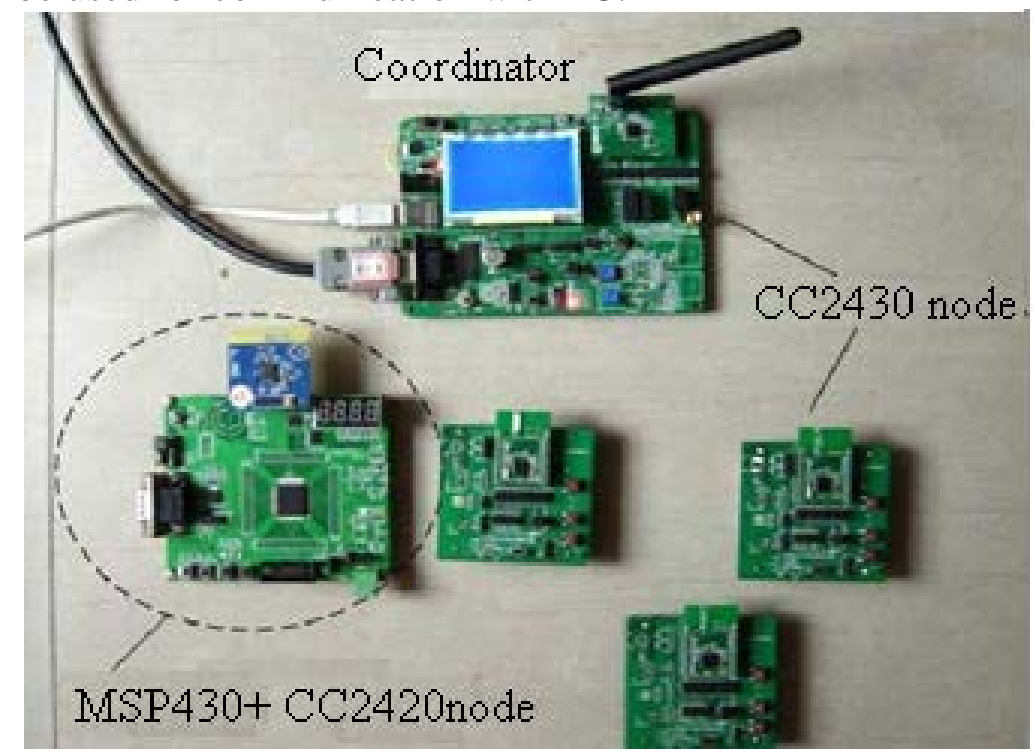

Fig.4: Node physical map and experimental platform

As shown in Figure 4, the node experimental platform mainly includes CC2430 full function node and CC2420 full function router, the MSPFG4618+CC2420 node and the rest of the node is as a terminal measuring equipment, and then using a ZigBee node connects PC machine through the serial port, in order to test the performance of the network. 


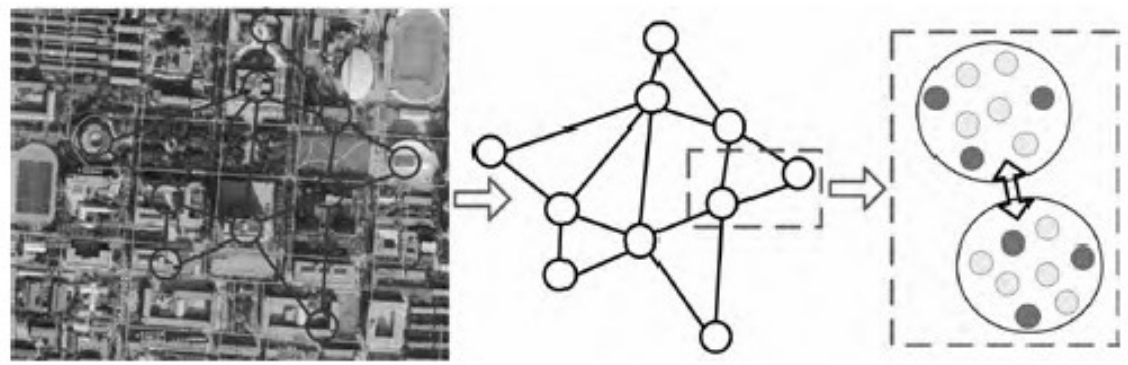

Fig.5: The movement project node distribution

Using the perceived direction of the nodes carry out nodes mobile application scene modeling based on position. As shown in Figure 5, each application scenarios have many user distribution intensive sites, and nodes in these places are gathered the group formation based on location spatial dispersion [13-15]. In order to improve the social relationship between groups, group can be connected by using these mobile nodes, opportunistic communication participates in the evaluation of sports, finally we can get the summary evaluation results of the project evaluation.

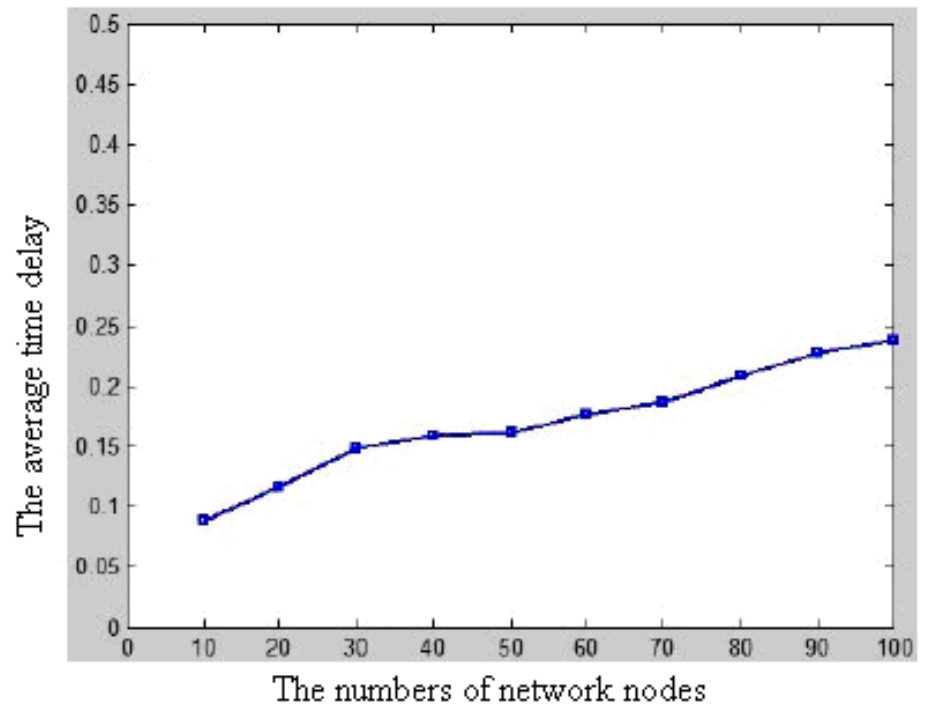

Fig.6: The curve of network average time delay

As shown in Figure 6, with the increase of the number of nodes of wireless sensor networks, network delay increases gradually. In below 50 nodes, delay variation is relatively stable, when the number of the node exceeds 50 , the delay increases gradually, and the maximum is not more than $30 \mathrm{~ms}$, to meet the needs of system design.

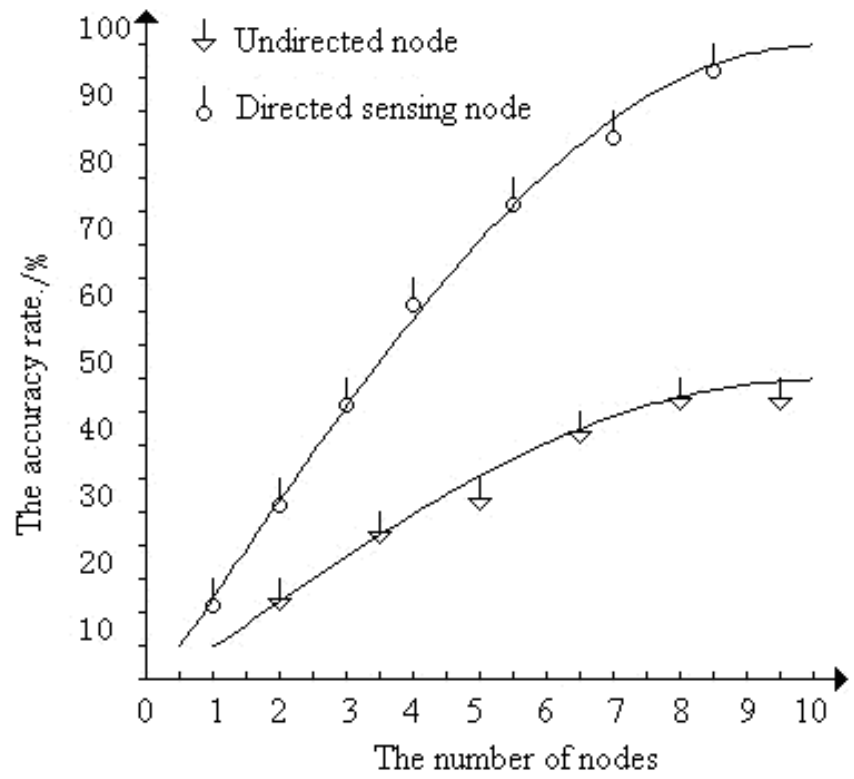

Fig.7: The results of node rationality distribution 
Figure 7 shows the rationality of analyzing the rural sports using nodes distribution [16,17]. In different algorithm, the accuracy of results are obtained that it can be seen from the chart, this paper designs the analysis accuracy rate of directional sensor nodes are more obvious than undirected node high, which is an efficient analysis method.

\section{Summary}

(1)Based on judgment relationship matrix and mobile node principle, this paper designs the mathematical model of directional aware wireless nodes, and using programming method allocates node cache space for the directional sensing node, which realizes the multi node interaction evaluation of rural sport project rationality analysis.

(2) In order to verify the validity and reliability of the model and algorithm, this paper designs the serial port expansion circuit and physical platform of wireless sensor network. Through the wireless communication experiments, this system can get the time delay characteristic curve and the analysis accurate rate curve of data transmission; the results show that the algorithm can improve the wireless network coverage, to enhance the transmission characteristics of wireless network.

\section{References}

[1] S. Gao, H.K. Zhang. Study of the delay constrained sensor network mobile sink path selection method. Chinese Journal of Electronics, 2011(4): 56-59.

[2] S. Gao, H.K. Zhang, H.S. Xu. The efficient data collection mechanism of sink trajectory fixed sensor networks. Journal of software, 2010(1): 136-137.

[3] J.Q. Chen, H.J. Liu, M.X. Zhu. The beneficial exploration of the rural sports public service supply in Jiangsu area. Sports and science, 2013(5): 98-99.

[4] X.P. Qin, Q. Xia, J. Wang, Q.S. Hu. Study of the rural sports public products supply under the non equilibrium. Journal of Beijing Sport University, 2013(4): 112-113.

[5] H. Zhang, Q. Chen. The responsibilities division of our public sports service different supply subjects. Journal of Guangzhou Sports University, 2013(2): 78-80.

[6] W.L. Yang, Y.M. Gao, Y. Liu. The basic theoretical framework construction and analysis of the sports public service system. Journal of Shenyang Sport University, 2012(6): 34-38.

[7] L. Liu, Q.S. Hu, A.Q. Liu, C. Fu. The research and construction of sports public service system in rural areas. Hubei sports science theory, 2012(5): 34-37.

[8] J. An, X.L Gui., W.D. Zhang, J.H. Jiang, J. Zhang. The social relationship cognition model of internet mobile perception. Journal of computer, 2012(6): 12-15.

[9] Q.S. Cai, J.W. Niu. The opportunity network time evolution map model based on edge independent evolution. Computer engineering, 2011(15): 23-26.

[10] X.F. Wang, Y.B. Liu. Overview of community structure algorithms in complex network. University of Electronic Science and technology, 2011(5): 49-50.

[11] S. Jiang, W.X. Wang, D.Z. Sun, Z. Li. Design of energy self-sufficient orchard information gathering wireless sensor network node. Journal of agricultural engineering, 2012(9): 45-48.

[12] W.X. Wang, X.W. Luo, D.Z. Sun, S. Jiang, G.K. Xiao. Design of tea information collection wireless sensor network node. Journal of agricultural engineering, 2011(5): 91-95.

[13] S. Zou, C.M. Liu, F.P. Li. Water environment monitoring system based on sensor network. Wireless sensor and micro system, 2010(9): 41-42.

[14] C.F. Wan, S.F. Du, L. Zhao. Research on sinus aquaculture environment monitoring system based on WSN. Agricultural machinery research, 2010(2): 81-84.

[15] M.D. Xue. The low power consumption temperature and humidity measurement system based on nRF905. Modern electronic technique, 2010(1): 34-38.

[16] S.X. Song. Analysis on rural sports public service supply path. Contemporary sports science and technology, 2012(15): 78-81.

[17] A.G. Zhou. The status and functions of the concept examine government from the sports public service. Sports science, 2012(5): 24-28. 